\title{
Background, design and conceptual model of the cluster randomized multiple- component workplace study: FRamed Intervention to Decrease Occupational Muscle pain - "FRIDOM"
}

\author{
Jeanette Reffstrup Christensen ${ }^{1 *}$, Thomas Viskum Gjelstrup Bredahl', Jenny Hadrévi², Gisela Sjøgaard ${ }^{1}$
} and Karen Søgaard ${ }^{1}$

\begin{abstract}
Background: Several RCT studies have aimed to reduce either musculoskeletal disorders, sickness presenteeism, sickness absenteeism or a combination of these among females with high physical work demands. These studies have provided evidence that workplace health promotion (WHP) interventions are effective, but long-term effects are still uncertain. These studies either lack to succeed in maintaining intervention effects or lack to document if effects are maintained past a one-year period. This paper describes the background, design and conceptual model of the FRIDOM (FRamed Intervention to Decrease Occupational Muscle pain) WHP program among health care workers. A job group characterized by having high physical work demands, musculoskeletal disorders, high sickness presenteeism - and absenteeism.

Methods: FRIDOM aimed to reduce neck and shoulder pain. Secondary aims were to decrease sickness presenteeism, sickness absenteeism and lifestyle-diseases such as other musculoskeletal disorders as well as metabolic-, and cardiovascular disorders - and to maintain participation to regular physical exercise training, after a one year intervention period. The entire concept was tailored to a population of female health care workers. This was done through a multi-component intervention including 1) intelligent physical exercise training (IPET), dietary advice and weight loss (DAW) and cognitive behavioural training (CBT).

Discussion: The FRIDOM program has the potential to provide evidence-based knowledge of the pain reducing effect of a multi component WHP among a female group of employees with a high prevalence of musculoskeletal disorders and in a long term perspective evaluate the effects on sickness presenteeism and absenteeism as well as risk of life-style diseases.
\end{abstract}

Trial registration: NCT02843269, 06.27.2016 - retrospectively registered.

Keywords: RCT, Worksite, Health promotion, Implementation, Maintained effect, Sickness presenteeism, Sickness absenteeism, Exercise, Diet, Cognitive behavioral training

\footnotetext{
* Correspondence: jrchristensen@health.sdu.dk

${ }^{1}$ Department of Sports Science and Clinical Biomechanics, University of

Southern Denmark, Campusvej 55, 5230 Odense M, Denmark

Full list of author information is available at the end of the article
} 


\section{Background}

The consequences of unhealthy lifestyle constitute a major public health challenge in Western countries [1-3]. Factors such as physical inactivity and poor diet, can lead to overweight or obesity and consequently musculoskeletal pain. It has large economic consequences for society, in general, and for workplaces and the individual worker, in particular, in terms of reduced productivity, long-term sickness absence and premature exit from the labor market [4-7]. Unhealthy lifestyle and long-term sickness absence are common in low education jobs such as health care workers, a predominantly female job group also characterized by low physical capacity in terms of low muscle strength and aerobic capacity, as well as high prevalence of musculoskeletal pain and overweight or obesity [8-11]. Health care work consists of many hours of walking and standing with a wide variety of physical activities such as cleaning and patient handling, often involving large physical demands and peak forces $[9,12]$. Studies suggest, that the high frequency of lifestyle diseases including musculoskeletal pain and sickness absence among health care workers, is caused by the mismatch of heavy work tasks and a low aerobic fitness level, low muscle strength and excessive body weight $[8,11,13-16]$.

In a physically heavy job, high body weight imposes a high biomechanical strain on joints and muscles and a large body frame may hinder optimal ergonomic working postures during patient handling. Thus, obesity is a focal risk factor closely associated with a large number of lifestyle diseases also including musculoskeletal pain $[17,18]$.

Changes in humoral factors such as an increase in triglycerides, low-density lipoprotein (LDL) and cholesterol relate to inactivity and obesity, and are well known as cardiovascular risk factors [19, 20]. However, also for musculoskeletal pain metabolic markers have for long been an issue of investigation. Recent studies have in myalgic muscle interstitial fluid presented a number of metabolites associated with muscle pain [21-24]. However, a possible relation between humoral factors and the pain related muscle metabolites linking obesity and musculoskeletal pain have not been studied.

In the last decades, workplace health promotion (WHP) programs have emerged to improve the personal health of employees and to reduce organizational health-related expenses $[25,26]$. A sizeable amount of evidence has accumulated towards the workplace as a promising setting for health promotion since it offers an efficient structure to reach large groups of people and makes use of natural social networks [26-28]. However, participation is required to ensure a clinical effect in the intervention. Adherence to work place exercise training is challenging and often displays a large number of drop-outs [29, 30]. However delivering WHP in working hours, paid by the workplace, lodges a possibility to a high participation to the WHP This should be supported by important factors such as leadership, organizational issues and instructors [31, 32]. Moreover, monotonous content, lack of inspiration and transferability to leisure time physical activity seem to be a barrier toward adherence [31].

This is supported by behavioral theory as e.g. the Self-Determination Theory (SDT) explaining the importance of autonomous motives, feeling of competence, control and relatedness for motivation and consequently adherence to a specific behavior [33]. Furthermore, the Theory of Planned Behavior explicit the need for perceived behavioral control (PBC) to enhance the possibility of progressing from intention to behavior or from work place physical exercise to regular leisure time exercise training [34, 35].

Educational level and occupational class is inversely associated with poor physical capacity and overweight or obesity, particularly among women [36, 37]. Since education and gender often are important factors in the stratification into certain occupational sectors, WHP have an opportunity to reach a specific target population and engage individuals who may otherwise face obstacles in participation in health-related activities [36-39]. WHP for health care workers therefore seem to be a legitimate attempt to reduce the growing health inequality in society by targeting this high-risk group.

The present protocol describes a FRamed Intervention to Decrease Occupational Muscle pain (FRIDOM). The main aim was to reduce neck and shoulder pain, secondary aim was to decrease lifestyle-diseases such as other musculoskeletal, metabolic-, and cardiovascular disorders. FRIDOM aimed eventually to decrease sickness presenteeism and sickness absenteeism. This was done through a multi-component intervention including 1) intelligent physical exercise training (IPET) [40], dietary advice and weight loss (DAW) and cognitive behavioural training (CBT). In a long term perspective the aim was to maintain participation to regular physical exercise training, beyond the 1 year intervention period. The entire concept was tailored to a population of female health care workers.

\section{Methods/Design}

The FRIDOM program was composed of four parts: 1) A planning phase according to intervention mapping principles [41], 2) a feasibility study to reveal issues of implementation and intervention content, 3) a randomized controlled trial (RCT), and 4) a maintenance period. Below these components are further detailed. 


\section{Intervention mapping and time frame}

The main focus of the intervention was on sustainable behavioural life-style changes, therefore, a key point was a detailed planning of all phases to ease the final maintenance of the introduced health enhancing activities after the 12 months supervised intervention. The intervention planning included a need assessment in order to facilitate participation and another crucial part was to establish a firm organizational support for the intervention. Therefore all stakeholders in the municipality from the top level management to the individual health care worker were informed about the project and invited to information meetings on the intervention content, organization and management in the planning phase. The detailed planning was based on the following premisses: 1) The municipality should own the intervention and allow resources from the municipality to the intervention logistics. 2) The organization participated in a steering and working group and should allow time for meeting activities in these groups. 3) All employees were given the possibility to participate in the intervention at or nearby their geographical workplace during paid working time. 4) The intervention content should appeal to the participants. 5) The health effect of the intervention content should be evidence based. 6) The design should allow evaluation of the effect on primary and secondary outcomes, and 7) the intervention content should be integrated in the general work environment already taking place at the workplace [41]. The intervention mapping started around summer time 2013 and the subsequent planning resulted in commencing the feasibility study in January 2014 and the RCT study in August 2014, as shown in Fig. 1. The figure also presents duration of the different phases together with the time points for the measurements taken.

\section{The FRIDOM-Feasibility study}

To prepare and facilitate the FRIDOM-RCT study a feasibility study was conducted which served several purposes: 1) To build confidence in the project internally in the municipality, 2) to allow a participatory approach, where experiences and suggestions from participants for improvement of the intervention content could be utilized in the final implementation in the RCT study, 3) to test if the intervention content planned for the RCT was practically suited for implementation in the municipality context, 4) to lean by and overcome organizational and practical barriers for participation and 5) evaluate both effectiveness and efficiency of the physical training program within the target group of health care workers.

Before the intervention in the feasibility study was initiated, all eligible employees were asked to fill out a screning questionnaire and participate in a baselinescrening-test. To allow for subsequent adjustment of the intervention content and mode of practical implementation, the feasibility study was timed approximal 7 months ahead of the RCT study, according to the same principal schedule as the RCT (Fig. 1). The maintenance period started January 2015 for the FRIDOMFeasibility study (Fig. 1).

\section{The FRIDOM-RCT study}

The FRIDOM-RCT was implemented as a single-blinded cluster randomized controlled trial (RCT) with a "stepped wedge" design (Fig. 1) [41, 42]. Content of the intervention had to be given repeatedly, and it was an important step to avoid changes in intervention delivery to the successive cohorts. In clinical intervention research, the RCT is considered the gold standard [43]. However, in WHP programs there appear to be three major obstacles: Firstly, the introduction of control clusters not receiving treatment may be considered unfair and can impair organizational commitment [42, 44]. Secondly, to maintain the intervention within all consenting employees, it was important that all employees were being offered the intervention at some point in time. Thirdly, from a workplace point of view, there could be a

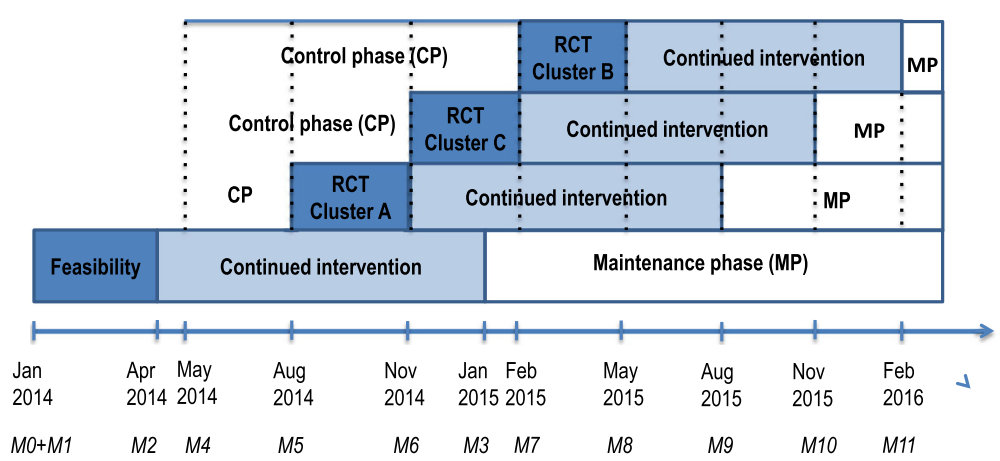

Fig. 1 Time frame of feasibility and RCT study. Legend: The screening test on all 348 participants is numbered MO. The tests within the FRIDOMfeasibility study are numbered M1-M3 and the tests within the FRIDOM-RCT are numbered M4-M11 
problem in implementing the program simultaneously to all consenting employees because of practical and logistical reasons [42, 44]. These three difficulties could all be overcome in the more feasible stepped-wedge design that enabled all consenting employees to receive the intervention.

\section{The FRIDOM-maintenance}

The primary goal in the FRIDOM program was to reduce neck-shoulder pain (Fig. 2). Secondary goals were to decrease sickness presenteeism and sickness absenteeism by decreasing lifestyle-diseases through a multi-component intervention of intelligent physical exercise training (IPET) [40], dietary advice and weight loss (DAW) and cognitive behavioural training (CBT). Finally it was a long term goal to implement the intervention elements as permanent activities at the workplace, maintained beyond the 1-year intervention period. Therefore, the activities offered at the workplace should include not only baseline consenters, but also gradually involve new employees, employees returning to work from maternity leave, long-time sickness absence, sabbatical leaves as well as employees changing their mind regarding participation.

The maintenance of the activities introduced in the intervention was facilitated by a comprehensive reorganizational change in working plans in order to integrate a permanent one weekly work hour for intervention activities in the normal working plans for all teams of health care workers. After the 1-year intervention, the weekly planned training hour within work time was maintained, but employees were expected to work time off at another time point.

\section{Study population}

\section{Participants}

The FRIDOM program was offered to the entire elderly care section in a medium size municipality in Jutland, Denmark. The health care section was divided in two main areas (RISOE and ROLI) with two different Area Managers. Each area was then divided into different centers for elderly people and retirement homes. These were spread out in five cities within the municipality. The cities are named Ebeltoft, Ryumgaard, Roende, Hornslet and Knebel. The main group of employees in the entire elderly care section was home care helpers (14 months of education) and home care assistants (21 months of education on top of the 14 months education as home care helpers) and in combination they are referred to as health care workers. All eligible personnel at the entire elderly care section (health care workers, nurses, activity staff, leaders, administrative staff and janitors) were invited to participate in the project to optimize motivation to comply with the intervention. Inclusion criteria were that participants: 1) were employed at the workplace for at least $15 \mathrm{~h}$ per week, 2) were permanently employed, or 3 ) had at least 12 months of work left before retirement. Exclusion criteria were long-term sick listed, pregnancy or working at a rehabilitation center, as these employees did not have work tasks within elderly care.

\section{Recruitment procedure}

Recruitment of eligible participants was based on the official list of staff that contained 496 employees (Fig. 3). Besides the list, the Area Managers were aware of 11 recently employed health care workers, and these were included as well, summarizing 507 employees on the elderly care section. The list was scrutinized excluding employees no longer employed, working less than $15 \mathrm{~h}$ a week, on either maternity or long-term sick leave. Additionally, 28 employees working in a separate rehabilitation center - all being either occupational- or physiotherapists - were not informed about the project and not offered participation. The remaining 448 employees were at the beginning of 2014 invited to information meetings during working hours where information about the FRIDOM program and a screening questionnaire were provided. A total of seven meetings were held to reach all eligible participants. Prior to the meetings, written information was distributed to the employees in a short information pamphlet. Managers supplied employees, who did not attend the information meeting with written information and a screening

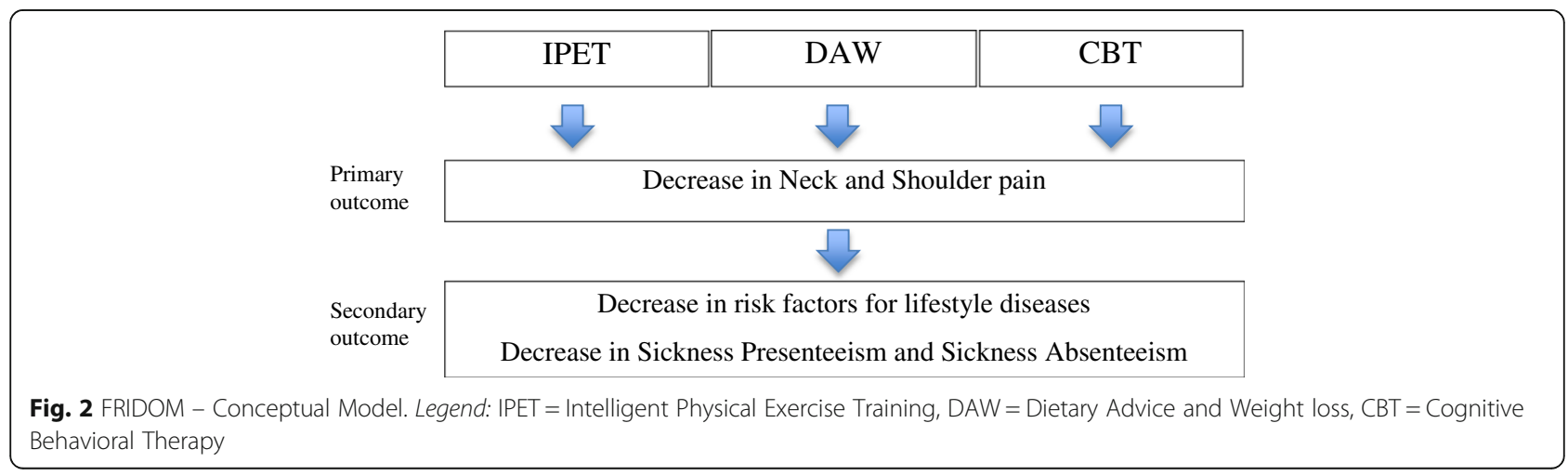




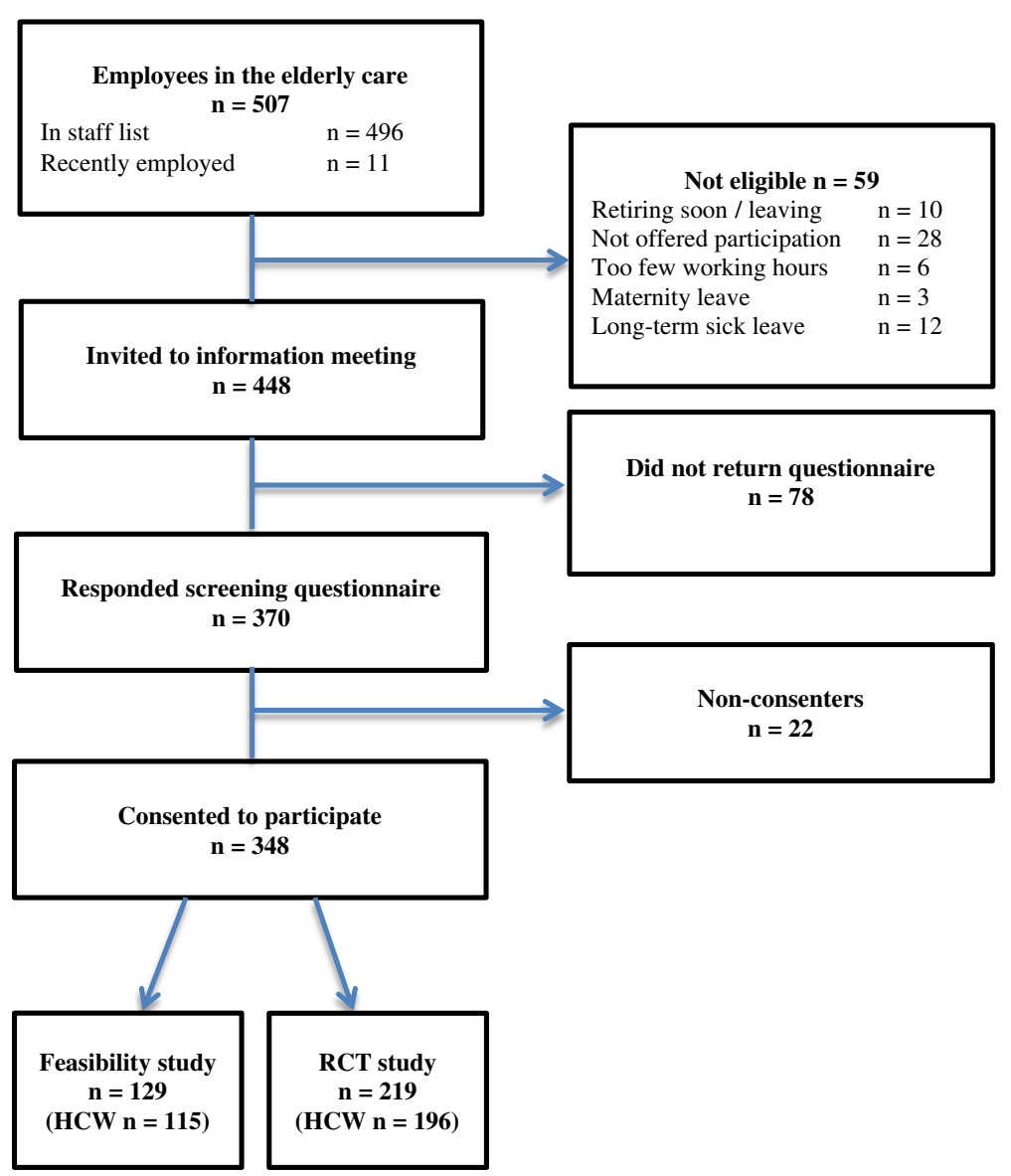

Fig. 3 Flow chart of employees. Legend: HCW = Health Care Workers

questionnaire. A total of 370 replied to the screening questionnaire and 348 consented to participate (Fig. 3). These were divided into 129 for the FRIDOMFeasibility study and 219 for the FRIDOM-RCT study.

\section{The FRIDOM-Feasibility study}

The feasibility study involved 129 participants and was conducted among employees working in RISOE area. This part included both health care workers, working in centers for elderly people and retirement homes (together home care facilities) and employees working in the elderly's private home in both day, evening and night shifts.

\section{The FRIDOM-RCT}

Randomization The RCT clusters of 219 participants were individually allocated to a team by the first author JRC, according to the following criteria for minimizing variation within a team: 1) Geographical location of the workplace, 2) working at center/home care facilities or in the elderlies private home, and 3) day or evening/ night shifts. This allocation resulted in 19 teams. To maximize motivation by social bonding with colleagues within a team, close relations with colleagues were taken into consideration. Subsequently, the 19 team were combined into three clusters of approximal same magnitude. In order to minimize contamination between clusters, priority was given to minimize variation in geographical location within a cluster. Additionally, the aim was to minimize variance of job exposure between clusters. Therefore balancing was perfomed between clusters regarding criteria 2 and 3 mentioned above, as well as ensuring a relatively even distribution of health care workers and nurses between the three clusters. This resulted in the three clusters composed as A: 5 teams from Roende and 2 teams from Ryumgaard, B: 5 teams from Hornslet, and C: 3 teams from Hornslet and 4 teams from Knebel. These tre clusters were randomized by author GS, using an opaque bag with the tree letters written on small paper cards. First letter drawn from the bag was $A$, then $C$, and finally $B$, which then defined the order of the clusters entering the stepped wedge design (Fig. 1).

Blinding The outcome assessors were blinded to the employee's cluster assignment. At follow-up testing, the employees were informed not to tell the outcome assessors 
what cluster they were assigned to. The outcome assessors were also trained to not discuss cluster allocation with the participants. All researchers and data analysts were blinded to cluster allocation. However, due to the content of the physical exercise training, participants and instructors could not be blinded to cluster allocation.

\section{Intervention content}

The 1-hour weekly multi-component intervention consisted of a combination of intelligent physical exercise training (IPET) including Power Breaks, dietary advice and weight loss (DAW) and cognitive behavioral therapy (CBT). An overview of the content over time is provided in Fig. 4.

\section{Intelligent physical exercise training (IPET)}

The principles for IPET were that the exercise training was adjusted to 1) occupational physical exposure, 2) physical capacity, 3) health status and 4) motivations and barriers. As FRIDOM targeted a homogeneous job group, the selection of exercises and training program was adjusted to the specific job demands of health care workers.

The physical exercise consisted of two elements: strength training exercises with elastic band as resistance (Power Breaks) and aerobic exercises. Four strength training exercises specifically targeting the neck and shoulder muscles were selected: Frontal raise, Lateral raise, Shrugs and Reverse flies (see Fig. 5). During the first month, $30 \mathrm{~min}$ of the one hour weekly session were devoted to familiarizing and correct performance of these exercises. This also included the choice of elastic band color and adjustment of length to provide the correct resistance of $12 \mathrm{RM}$ and how to increase this to 10 RM. During months 2 and 3 the concept of a daily power break including $10 \mathrm{RM}$ with 2 of the 4 exercises was introduced. The daily Power Breaks were now expected to be performed in addition to the weekly training sessions either during work time or at home. Aligned with the normalization process theory, participants were told to find an everyday suitable 5 min power

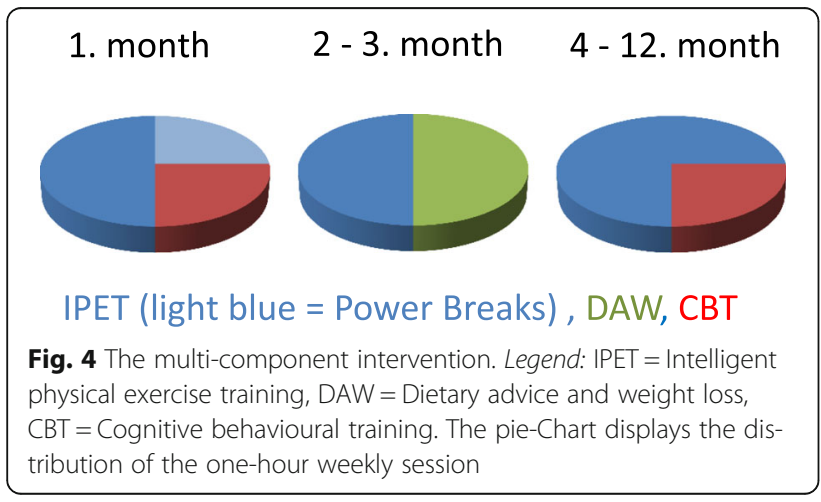

break. Most optimal at the exact same time a day, in order to make the power breaks a new every day habit [45]. The aerobic exercises were inspired by the protocol developed in a previously workplace study [11]. The aerobic activities aimed at a level of $70 \%$ aerobic capacity including warm up, circuit training and playing activities. In the first weekly sessions, aerobic activities were introduced in addition to the power breaks and with intensities around $50 \%$ aerobic capacity. Over the first month the duration of the aerobic activities were gradually increased. In the second and third months, intensity in the aerobic exercises targeted a level of $70 \%$ aerobic capacity to result in high calorie consumption to increase aerobic capacity and support weight loss. The last 9 months, focus was on being more physically active in leisure time - introducing outdoor power walks, hiking, running, biking or trying out different sports offered in local sport clubs. Complete lists of leisure time physical exercise possibilities in the municipality were presented to the participants. In all training clusters, a motivation was that the participants should decide and agree upon which aerobic exercise activities they would focus on. Four out of the 46 scheduled exercise sessions were replaced by visits to physical activity possibilities in the municipality. This was done to help the participants feel autonomous as highlighted by SDT [33] and to overcome the intention-behavior gap as explained by TPB [35] and to enhance motivation by introducing fun and playful elements to the IPET training.

\section{Dietary advice and weight loss (DAW)}

All participants were guided to ensure a healthy diet and prevent weight gain by adjusting courses according to the Danish Dietary recommendations, e.g. reduction of refined sugar, reduction of especially saturated fat, carbohydrates from primarily fiber-rich sources, and $600 \mathrm{~g}$ of fruit and vegetables per day. These guidelines were also consistent with new findings regarding weight loss [46]. Participants, who aimed to loose weight were encouraged to fill out a four days dietary record providing information on dietary preferences [47]. For each individual the resting metabolism was calculated, based on gender, age and weight and multiplied by a Physical Activity Level factor (PAL) of 1.8 to estimate the daily energy requirement [48]. $\sim 1.000 \mathrm{cal}$ were subtracted from the estimated daily energy requirements and the resulting value was used as individual calorie prescription. Individual guidance was provided for all meals with specific calorie amounts adjusted to suit the individual calorie prescription and dietary preferences. As shown in Fig. 4, the DAW-course was carried out during the second and third month, occupying approximately $30 \mathrm{~min}$ of the weekly session. From the fourth to the twelfth month, every session began with a weight check and individual dietary advices for those who still wanted to lose or maintain weight. 


\begin{tabular}{|c|c|c|c|c|c|c|c|}
\hline Week & Date & \multicolumn{2}{|c|}{ Exercise } & Repititions & Sets & Number of windings & Band color \\
\hline \multirow[t]{2}{*}{42} & 13.10 .15 & A & $\begin{array}{l}\text { Lateral } \\
\text { raise }\end{array}$ & \multirow{2}{*}{$\begin{array}{l}10 \text { repetitions } \\
\text { at } 10 \mathrm{RM}\end{array}$} & 3 & 1 & Red \\
\hline & & B & Strucks & & 3 & 3 & Green \\
\hline \multirow[t]{2}{*}{42} & 14.10 .15 & $\mathrm{C}$ & $\begin{array}{l}\text { Front } \\
\text { raise }\end{array}$ & \multirow{2}{*}{$\begin{array}{l}10 \text { repetitions } \\
\text { at } 10 \mathrm{RM}\end{array}$} & 3 & 2 & Red \\
\hline & & $\mathrm{D}$ & $\begin{array}{l}\text { Reverse } \\
\text { flies }\end{array}$ & & 3 & 1 & Blue \\
\hline
\end{tabular}

Fig. 5 Examples of strengthening exercises performed as power breaks. Legend: RM = Repetitions Maximum

\section{Cognitive behavioral training (CBT)}

The CBT training was developed in a previous research project by Christensen et al. [11]. In short it was based on Linton's model for coping with chronic pain modified to address discomfort during weight loss and to support a change to a more physically active lifestyle $[49,50]$. The CBT was provided as group discussions and followed a specifically tailored guideline, containing exercises such as pro-and-con schemes and positive thinking strategies with homework between each session. During the first month, about 15 min of the weekly sessions were used on CBT (Fig. 4), helping the participants to make realistic weight loss targets and to support a change to a more physically active lifestyle. From the 4th to the 12th month of the intervention, about $15 \mathrm{~min}$ were spent on CBT in the weekly sessions. Focus was to reflect on dysfunctional attitudes and coping behaviors on how to continue healthy behaviors concerning both weight loss or weight maintenance and maintaining a physically active lifestyle. To support the aim of participant adherence to workplace physical exercise and regular leisure time physical exercise training, motivational initiatives based upon Self-Determination Theory (SDT) were included. To influence the feeling of autonomy, competence and control, the participants filled in a questionnaire expressing their wishes for leisure time physical exercise training after one month of intervention. Within the same questionnaire the participants were asked to describe possible barriers for their participation in leisure time physical exercise training but also possible solutions to overcome barriers. As a structured part of the RCT intervention and the cognitive training, the participants, discussed in groups on how to overcome barriers. These discussions were done to provide the participants the possibility to feel autonomous and competent and also to increase the relatedness to colleagues, Furthermore, motivational factors for their continued adherence were included in the discussion. During month two and three, the motives and barriers from the questionnaire concerning leisure time physical exercise were discussed again within the training clusters.

\section{Modifications from FRIDOM-Feasibility to the FRIDOM-RCT}

Based on feedback from the participants a few changes were done before the intervention of the FRIDOM-RCT started. DAW and Power Breaks were introduced from the very start of the intervention. While the power breaks were to be performed in teams in the feasibility study, this was not feasible to integrate in the working days schedule. Instead, an individual dairy log with a detailed instruction was developed and handed out. Power Breaks could then be performed during workday or in leisure time, when the individual found it convenient. In the FRIDOM-Feasibility, it was an organisational challenge to arrange the weekly FRIDOM sessions at the workplace. Health care workers often change work days and work shifts, and the steering group decided that it was not realistic to constantly adjust work schedules to fit the FRIDOM intervention. Therefore, it was accepted that participation rate was lower due to vacation, days off or work pressure. Further a burn in period ( $\sim 3$ month) was added to the FRIDOM-RCT allowing for information and planning of the logistic to incorporate the intervention.

\section{Instructors}

Due to Danish legislation, all employees were entitled to six weeks vacation. Thus, weekly training sessions were only carried out in 46 weeks and were all supervised with instructors. Most sessions took place at the workplace, but in 3-4 sessions, the training team visited local sports clubs in order to break barriers to leisure time exercise. The instructors were required to have a background as bachelors in sports, physiotherapy or public 
health. Every training team was supposed to be supervised with the same instructor during the entire year. During vacation or sickness in the instructor group, instructors would deputiz for each other, and no sessions were to be canselled. Instructors were paid from FRIDOM fundings in the intervention year.

\section{Outcome measures}

Outcome measures were collected in four different categories: (Categorie 1) data drawn from workplace registrations, (categorie 2) questionnaires, (categorie 3) physiological tests and (categorie 4) adherence. Besides the physical tests, the physiological tests included blood samples of blood sucker, cholesterol and triglycerid. Categorie 1-4 tests were collected on all participants, in either the FRIDOM-feasibility or the FRIDOM-RCT study. At some timepoints, a full packages of each category were collected (Large test), while at other timepoints, only a subsets were collected (Mini test). Besides the Large tests and the Mini tests, a screning test was initially conducted. As a nested study, additional blod samples and objective heart and physical activity measures were collected on a smallere representative subsample.

With reference to Fig. 1, the outcome measures are specified for each test. The screening test is numbered M0. The FRIDOM-feasibility test measures are numbered M1-M3 and the FRIDOM-RCT test measures are numbered M4-M11.

In January 2014 (M0), all 348 participants participated in a screning test and filled out a screning questionnaire. Shortly after, the feasibility cluster $(\mathrm{n}=129)$ was baselinetested with a Large test in the categories 1-3 (M1). In April 2014 (M2), the feasibility cluster had a Large test at follow up, as they also had after one-years intervention, in January 2015 (M3).

In May 2014, the 219 RCT participants were baselinetested with a Large test (M4) in the categories 1-3. In August 2014, the intervention of the first RCT-cluster commenced (M5), the second cluster in November 2014 (M6) and the third in February 2015 (M7) (Fig. 1). At each of these timepoints, and also in May 2015 (M8), the Mini test were performed on all three RCT-clusters and additional blod samples and objective heart and physical activity measures were collected on a smallere representative subsample only. The Mini tests, including objective heart and physical activity measures but without additional blod samples, continued within all three RCT-clusters every third months, in August 2015 (M9), November 2015 (M10) and February 2016 (M11). These tests were conducted as Mini tests after 3, 6 and 9 months intervention within each RCT-clusters, but as Large tests after 12 months intervention (Fig. 1).

\section{Workplace registrations (Category 1 )}

Information about age, sex, job seniority, weekly working hours, work shift (day shift, night/evening shift), care center or homecare, job type (home care helpers, home care assistants, nurses, leaders and administrative staff or other job categories) and educational level (unskilled, low skilled - $<2$ years of education - and high skilled $-\geq 2$ years of education) were gathered from the workplaces own registration on their employees.

Information on sickness absenteeism was retrieved from a local database maintained by the executive director at the workplace. The records listed the beginning and the end dates of each sickness absence spell for each employee 12 months before intervention start and until the end of the 12 months intervention. Number of spells and days for each month were registered. The number of days with sickness absence represented workdays only. Maternity leaves and absences attributable to caring for a child were not included in the sickness absence registrations.

\section{Questionnaire (Category 2)}

The FRIDOM program consisted of three different questionnaires. 1) A Large questionnaire given at baseline and followed-up after 1 year within each RCT-cluster, 2) a Mini questionnaire to be completed every third months, and finally 3 ) a Screening questionnaire distributed before randomization in January 2014 (Fig. 1). The Large questionnaire involved lifestyle behaviour and health (smoking, alcohol consumption, medicine use and sleeping behaviour), dietary intake (fruit, vegetables, fish, sugar and fat), physical activity during leisure time [51,52], self-rated health and Stress-Energy Scale, SF-36 [53, 54], physical resources with five items: aerobic fitness, strength, endurance, flexibility and balance/coordination, readiness to change [55, 56], Cohens Perceived Stress Scale (PSS) [57], chronic diseases, Standardized Nordic Questionnaires of musculoskeletal symptoms last seven days and pain intensity on a numeric rank box scale [58], general self-efficacy [59], Tampa Scale of Kinesiophobia [60], Work ability Scale [61], perceived physical exertion [62], productivity by a single item in the World Health Organization Health and Work Performance Questionnaire (HPQ) [63] and Quantity and Quality of work [64].

The Mini questionnaire every third months consisted of eight questions, included the Standardized Nordic Questionnaires for the analysis of musculoskeletal symptoms last 7 days and pain intensity [58], physical capacity with five categories: aerobic fitness, strength, endurance, flexibility and balance/coordination [65] as well as readiness to change $[55,56]$ and general self-efficacy [59].

The Screening questionnaire consisted of a subset of items from the Large questionnaire. The purpose was to evaluating reach of the intervention within the RE-AIM framework [66], questions were selected to compare 
important factors related to family, health and pain status for consenters and non-concenters and responders and not-responders. Pain was measured by Standardized Nordic Questionnaires for the analysis of musculoskeletal symptoms last 12 months [58].

\section{Physiological tests (Category 3)}

The FRIDOM program included three different testbatteries. A large test-battery measured at baseline and after one year, a Mini test every third months and a Screening test before randomization. All participants were tested with a Large test at baseline and after 1 year of intervention. For some of the tests with physical strain, some exclusion criteria were used: elevated blood pressure [67], angina pectoris, heart or lung prescription medication, current or previous serious illnesses and traumas, herniated disc, tennis elbow, golf elbow, carpal tunnel syndrome, significant level of musculoskeletal pain at the time of the test and pregnancy.

The large test lasted one hour and consisted of anthropometrical, health-related and physical capacity measures specified as the following. Height was measured to the nearest $\mathrm{mm}$ without shoes. Body weight was measured wearing light clothes, but without socks and shoes. One kilogram was subtracted from the weight measure to compensate for clothing. BMI was calculated as BMI = body weight $/$ height ${ }^{2}$. References to BMI was according to the standardized classification of WHO: $<25 \mathrm{~kg} / \mathrm{m}^{2}=$ normal weight, $25-30 \mathrm{~kg} / \mathrm{m}^{2}=$ overweight, $\geq 30 \mathrm{~kg} / \mathrm{m}^{2}=$ obese [68]. Body Fat was measured using a bio impedance device (TANITA SC-330), which was set to 'standard' while body frame and the participant's age, height and gender were entered. Waist circumference was measured over the umbilicus standing up and with clothes on, using an ergonomic circumference measuring tape (Seco 203 Girth measuring tape) and clothes thickness was noted. Blood pressure iwas measured in seated position after $10 \mathrm{~min}$ of rest with an electronic blood pressure monitoring device (Artsana CS 410). Three measurements were performed one minute apart and an average calculated as in Appleyard M 1989 [67]. Aerobic fitness was measured with the Wattmax test using a Monark E327 bicycle ergometer and a pulse belt and was carried out according to Andersen LB 1995.

Isometric maximal voluntary strength was measured with a validated standardized setup [69], measuring maximal voluntary handgrip, shoulder elevation, back flexion and extension force [70]. The participants performed a minimum of three attempts with steady increasing force to reach maximum within $3-5 \mathrm{~s}$. The test was repeated until a maximal of five contractions if the last attempt showed a more than $5 \%$ increase. The participant rested at least $30 \mathrm{~s}$ between each attempt. The maximal attempt was recorded for further analysis.
Standardized verbal command and encouragement was given to maximize the effort. Handgrip in both hands was measured using a grip strength measurer (Jamar) [71]. Shoulder elevation was measured with a Bofors dynamometer with the subject seated erect in a chair with legs hanging freely, arms hanging along the side and head facing forward. The distance from pressure point to sternoclavicular joint was measured as the moment arm [72]. Back flexion and extension were measured with the subject standing, facing/backing onto beam and support plate at the spina iliaca anterior superior. The Bofors dynamometer was fixed to pull horizontal with a belt positioned at the vertical level of $\mathrm{m}$. deltoid insertion on the humerus. The distance from the belt to a line through the crista iliaca and lumbalcolumna (L4L5 level) was measured for the moment calculation [73]. Balance test was performed as an unilateral stance test with eyes open and participants were instructed to look directly ahead at a black spot placed approximately $2 \mathrm{~m}$ in front of them at eye height. The participants stood on the dominant foot (defined as the foot used for standing while kicking a ball) with the big toe of the non-dominant foot leaning against the medial malleolus of the dominant foot. The test was performed for $30 \mathrm{~s}$ [65]. Each participant was allowed three trials. If all three trials resulted in loss of balance before ending the test, the classification was recorded as failed. Blood samples were obtained from the participants as fasting mornings samples. One set of samples was for analysis of plasma using heparin prepared tubes, centrifuged and stored in room temperature until analyzed. Another set of serum samples were sampled for metabolomics analysis and handled in accordance with recommendations for accurate metabolite handling followed by gas chromatography mass spectrometry analysis [74]. Metabolomics analyses were only analyzed in a selected subsample before and after the first 3 months of the intervention in the RCT study, (i.e. M5 and M6 for cluster A, M6 and M7 for cluster C, and M7 and M8 for cluster B (Fig. 1)).

These were stored on ice water for 5-10 min, followed by $5 \mathrm{~min}$ of centrifugation at temperature-controlled conditions. Serum was pipetted and stored in three tubes and put on dry ice before transportation and finally stored in $-80^{\circ}$. The plasma samples were analyzed for standard clinical measures of metabolites: highdensity lipoprotein (HDL), low-density lipoprotein (LDL), total cholesterol, and glucose at accredited hospital laboratories.

The Mini tests lasted $15 \mathrm{~min}$ and included: blood pressure, body weight, BMI, body fat, waist circumference, and balance test.

The Screening test lasted $3 \mathrm{~min}$ and included information's on weight, BMI and body fat collected with the same procedures as described within the large test. 


\section{Adherence (Category 4)}

The instructors registered participation in the 46 weekly multi-component training offered at the workplace. Performance of daily five minutes exercise with elastic bands (Power Breaks), color of the Thera band, and number of windings [1-3] were reported in a training diary (Fig. 5). The training diaries were collected every third months, and handed out so the dates in the diary were consistent with the next three months.

\section{Additional measures in a nested subsample Heart and physical activity}

Diurnal measurements consisting of physical activity and electrocardiogram were performed on a random subset of participants at M5, M6, M7 and M8 (Fig. 1). At each timepoint measurements included approx. 25 participants starting the active intervention and approx. 25 participants in waiting clusters or having finalized the intervention. Actigraph GT3X+ accelerometers (www.actigraphcorp.com) were used to quantify physical activity and was worn for 4 days. The monitor determined accelerations in three directions with a frequency of $30 \mathrm{~Hz}$. Accelerations were sampled with a precision of 12 bit, with a dynamic range of $\pm 6 \mathrm{G}$ and stored as raw data. The accelerometer was mounted on the right thigh midway on the line between the iliac crest and the top of patella and orientated with the $\mathrm{x}$-axis pointing downwards, $\mathrm{y}$-axis horizontally to the left and $\mathrm{z}$-axis horizontally forward. To insure firm position on the skin we used $3 \mathrm{M}$, Hair-Set, double sided adhesive tape and Fixomull, BSN medical. The Actigraphs were then covered with waterproof folio, initialized for recording and data were before analysis downloaded using commercial software (Actilife version 5.5). Physical activity was subsequently quantified as time in sitting, standing and walking using custom build software (Acti4).

Electrocardiogram for estimation of heart rate and heart rate variability was recorded using e-Patch data loggers (AMORS, AMS 3000 Delta Technology) initialised with gender and age using the commercial software Hasimed. The logger was mounted in a disposable adhesive pre-gelled electrodes patch, placed on sternum, $1 \mathrm{~cm}$ below clavicula. Recordings with a sensitivity of $0.25 \mathrm{mV}$ covering $24 \mathrm{~h}$ of EKG stored with a frequency of $2000 \mathrm{~Hz}$. Data were downloaded using the commercial software Hasimed and the off-line detection of the timing of the R-peaks in the QRS complex allowed for calculation of heart rate and heart rate variability.

During the diurnal measurements, participants were asked to follow their normal every-day schedule life and to note in a paper diary, working hours, sleeping and waking time, and if any of the monitors were removed before scheduled.

\section{Statistics}

Analyses of outcome were performed both on the entire population and on the health care target population. The primary outcome measure was questionnaire based self-reported duration and intensity of neck pain with all other outcome measures considered secondary outcomes. For the Feasibility study the pre-post test were analyzed using paired $\mathrm{t}$-test and repeated measures. For the RCT differences between the three clusters at baseline were to be tested with Pearson's $x^{2}$ for distribution in sex, education (health care workers), current smoking status and the dichotomized parameter for musculoskeletal symptoms in neck, shoulders, upper- and lower back. All other parameters were to be tested with a Student's t-test. Changes over the 3 months intervention periods were compared with the changes over the corresponding 3 months control periods in an ANCOVA analysis in accordance to the intention-to-treat principle, i.e. all randomized participants were included in the analyses with missing values substituted with carried forward or backwards measured variables. Clusters, age and the investigated value at baseline were included as covariates. All results were given as mean (SD). $P<0.05$ were considered statistically significant. SPSS statistics 23 was used for the statistical analysis.

In order to account for the combined effect within and between subjects and repeated measurements on the same subject, linear mixed effects models were used. Mixed effects models account for the inter-correlation of repeated measures. In this study, treatment as a categorical variable with two levels comparing intervention measurements with control measurements was included in the models as a fixed effect. Inter-correlation of repeated measures was included as a random effect. The analyses were further adjusted for baseline values of the respective outcome in a second model. Additionally, we used likelihood ratio tests to compare the models with and without a covariance structure. Analyses were based on the intention to treat principle including all eligible participants without imputations since mixed effects models inherently account for missing values.

Sample size for the RCT part was estimated using the method described by Woertman \& colleagues for sample size calculation [75]. The study was powered to detect a between-groups mean difference in the primary endpoint of 1 point in neck and shoulder pain intensity, which has been considered a relevant change in the workplace context in terms of risk of sickness absence. The variance was set to 2.0 based on results from a $\mathrm{Da}$ nish study [16] within a similar population, $\alpha$ to 0.05 , power to 0.8 , and an intra-cluster correlation coefficient to 0.05 in the three steps. We calculated that we needed 82 participants in total and with a dropout rate of up to $50 \%$ we planned to recruit at least 160 . However, 
to support implementation and support the workplace ownership the study design aimed to include all volunteer employees. This approach would improve generalization of the study findings to municipalities who would introduce similar health enhancing initiatives for their employees.

\section{Discussion}

The FRIDOM program was designed to provide evidence-based knowledge of the pain reducing effect of a multi component WHP among a female group of employees with a high prevalence of musculoskeletal disorders. Musculoskeletal pain as well as overweight may be barriers for engaging in physical activity that can prevent life style diseases. Therefore the present study in a short-term perspective focus on physical activity to reduce neck shoulder pain and to support weight loss.

In a long term perspective health care workers with high physical demands present with a high risk for physical deterioration seen as low physical fitness, poor general health status, a high degree of sickness presenteeism and sickness absenteeism. Therefore the present study is designed to facilitate the implementation of the physical activities into normal daily life routines beyond the one-year intervention period.

Based on the IPET concept, we have in former studies successfully improved health among employees in a number of different job categories ranging from physically inactive to low, moderate, and finally, heavy physical work [40, 76]. The FRIDOM program specifically build on the former IPET results among health care workers adding a specific focus on influential factors for adherence to work place exercise training such as supporting organizational changes and facilitating an integrated gradual transfer from work place physical exercise to regular leisure time exercise training.

Other strengths of the FRIDOM program is the pragmatic design using a stepwise inclusion of all employers allowing for a strict effect evaluation in a 12 months perspective but still supporting a high degree of implementation at the workplace allowing for a long term effect. Another strong feature is the large feasibility study that as part of the intervention mapping allows a participatory approach to optimize integration of the intervention activities into the daily work routines and increase the sustainability beyond the intervention period.

All together the specific features of the FRIDOM program will provide evidence-based knowledge of the pain reducing effect of a multi component WHP among health care workers and in a long term perspective affect sickness presenteeism and absenteeism as well as risk of life-style diseases.

\section{Additional file}

\section{Additional file 1: SPIRIT 2013 Checklist. (DOC 437 kb)}

\begin{abstract}
Abbreviations
CBT: Cognitive behavioral training; DAW: Dietary advice and weight loss; FRIDOM: FRamed Intervention to Decrease Occupational Muscle pain; HCW: Health care workers; HDL: High density lipoprotein cholesterol; IPET: Intelligent physical exercise training; LDL: Low density lipoprotein cholesterol; RCT: Randomized controlled trial; WHP: Workplace health promotion
\end{abstract}

\section{Acknowledgements}

We kindly thank all the participating workplaces at Syddjurs Municipality and their employees, without whom this study would not have been possible.

\section{Funding}

The Ministry of Culture Committee on Sports Research, Denmark, The Danish Arthritis Society and the Simon Fougner Hartmann's Family foundation supported this study. The FRIDOM study has undergone peer-review by the funding body. The two largest grants are from governmental funding's - The Ministry of Culture Research Committee is an advisory committee for the Innovation Fund. The Ministry of Culture Research Committee assesses whether the projects are worthy of support, and the research assessment is subsequently approved by the Innovation Fund. There are no fundings from commercial organazations.

Availability of data and materials

Not applicable.

\section{Authors' contributions}

JRC, TB, GS and KS concepted the FRIDOM program and designed the multi-component intervention. JH contributed with specific methodological knowledge on the nested design and the advanced analysis of blood samples. JRC was responsible as the leader of the FRIDOM program, the workplace, participant recruitment, intervention, test sessions and data collection. KS acted as the guarantor. The statistical analyses were agreed upon between the authors. JRC drafted the first version of the manuscript, all authors contributed in writing of specific sections and finally read and approved the final manuscript before submission.

\section{Competing interests}

The authors declare that they have no competing interests.

\section{Consent for publication}

Not applicable.

\section{Ethics approval and consent to participate}

The FRIDOM program was approved by the The Regional Scientific Ethical Committees for Southern Denmark - number S20130157 and was conducted in accordance with the Helsinki declaration. The Additional file 1 was thoroughly reviewed and the guidelines were followed. The feasibility and RCT parts were retrospectively registered in the Clinical Trial Registration number NCT02843269.

To reach all eligible participants, 11 introductory one-hour meetings were held in the end of 2013. After the meetings, the employees filled out a screening questionnaire including the question "Do you want to participate in the FINALE-study?". Employees, who did not attend the meetings, were given written information and screening questionnaires from their closest manager. Questionnaires and possible consent were returned in a sealed envelope. At the baseline test, all employees who wanted to participate filled out declaration of consent. The participants took a copy home, and the originals were placed in a locked file cabinet at the university. The participants were informed, both verbally and in writing, that their consent could be redrawn at any time and without any questions asked.

\section{Author details}

'Department of Sports Science and Clinical Biomechanics, University of Southern Denmark, Campusvej 55, 5230 Odense M, Denmark. ²Department of Community Medicine and Rehabilitation, Sports Medicine, Umeå University, 90187 Umeå, Sweden. 


\section{Received: 2 August 2016 Accepted: 8 October 2016} Published online: 24 October 2016

\section{References}

1. Global status report on noncommunicable diseases 2010. Geneva: World Health Organization; 2011.

2. Phillips $P$. The rising cost of health care: can demand be reduced through more effective health promotion? J Eval Clin Pract. 2002:8:415-9.

3. Prentice AM. The emerging epidemic of obesity in developing countries Int J Epidemiol. 2006;35:93-9.

4. Organisation WH. The World health report 2002. Reducing risks, promoting healthy life. World health Organisation; 2002.

5. Rongen A, Robroek SJ, van Lenthe FJ, Burdorf A. Workplace health promotion: a meta-analysis of effectiveness. Am J Prev Med. 2013;44:406-15.

6. Wright DW, Beard MJ, Edington DW. Association of health risks with the cost of time away from work. J Occup Environ Med. 2002;44:1126-34.

7. Stewart WF, Ricci JA, Chee E, Morganstein D, Lipton R. Lost productive time and cost due to common pain conditions in the US workforce. JAMA. 2003;290:2443-54

8. Pohjonen T. Age-related physical fitness and the predictive values of fitness tests for work ability in home care work. J Occup Environ Med. 2001:43:723-30

9. Torgen M, Nygard CH, Kilbom A. Physical work load, physical capacity and strain among elderly female aides in home-care service. Eur J Appl Physio Occup Physiol. 1995;71:444-52

10. Andersen LL, Clausen T, Burr H, Holtermann A. Threshold of musculoskeletal pain intensity for increased risk of long-term sickness absence among female healthcare workers in eldercare. PLoS One. 2012;7:e41287.

11. Christensen JR, Faber A, Ekner D, Overgaard K, Holtermann A, Sogaard K Diet, physical exercise and cognitive behavioral training as a combined workplace based intervention to reduce body weight and increase physical capacity in health care workers - a randomized controlled trial. BMC Public Health. 2011:11:671.

12. Ono $Y$, Lagerstrom $M$, Hagberg $M$, Linden A, Malker B. Reports of work related musculoskeletal injury among home care service workers compared with nursery school workers and the general population of employed women in Sweden. Occup Environ Med. 1995;52:686-93.

13. Andersen $J H$, Haahr JP, Frost P. Risk factors for more severe regional musculoskeletal symptoms: a two-year prospective study of a general working population. Arthritis Rheum. 2007;56:1355-64.

14. McBeth J, Jones K. Epidemiology of chronic musculoskeletal pain. Best Pract Res Clin Rheumatol. 2007;21:403-25.

15. Waddell G. Preventing incapacity in people with musculoskeletal disorders Br Med Bull. 2006;77-78:55-69.

16. Rasmussen $C D$, Jorgensen MB, Clausen $T$, Andersen LL, Stroyer J, Holtermann A. Does self-assessed physical capacity predict development of low back pain among health care workers? A 2-year follow-up study. Spine. 2013;38:272-6.

17. Walker GE, Marzullo P, Ricotti R, Bona G, Prodam F. The pathophysiology of abdominal adipose tissue depots in health and disease. Horm Mol Biol Clin Investig. 2014;19:57-74

18. Tsuritani I, Honda R, Noborisaka Y, Ishida M, Ishizaki M, Yamada Y. Impact of obesity on musculoskeletal pain and difficulty of daily movements in Japanese middle-aged women. Maturitas. 2002;42:23-30.

19. Roberts CK, Hevener AL, Barnard RJ. Metabolic syndrome and insulin resistance: underlying causes and modification by exercise training. Compr Physiol. 2013;3:1-58.

20. Dandona P, Aljada A, Chaudhuri A, Mohanty P, Garg R. Metabolic syndrome: a comprehensive perspective based on interactions between obesity, diabetes, and inflammation. Circulation. 2005;111:1448-54.

21. Gerdle B, Ghafouri B, Ernberg M, Larsson B. Chronic musculoskeletal pain: review of mechanisms and biochemical biomarkers as assessed by the microdialysis technique. J Pain Res. 2014;7:313-26.

22. Hadrevi J, Ghafouri B, Sjors A, Antti H, Larsson B, Crenshaw AG, et al Comparative metabolomics of muscle interstitium fluid in human trapezius myalgia: an in vivo microdialysis study. Eur J Appl Physiol. 2013;113:2977-89.

23. Hadrevi J, Bjorklund M, Kosek E, Hallgren S, Antti H, Fahlstrom M, et al. Systemic differences in serum metabolome: a cross sectional comparison of women with localised and widespread pain and controls. Sci Rep. 2015:5:15925
24. Rosendal L, Larsson B, Kristiansen J, Peolsson M, Sogaard K, Kjaer M, et al. Increase in muscle nociceptive substances and anaerobic metabolism in patients with trapezius myalgia: microdialysis in rest and during exercise. Pain. 2004;112:324-34

25. Conrad P. Who comes to work-site wellness programs? A preliminary review. J Occup Med. 1987:29:317-20.

26. Robroek SJ, van Lenthe FJ, van Empelen P, Burdorf A. Determinants of participation in worksite health promotion programmes: a systematic review. Int J Behav Nutr Phys Act. 2009;6:26.

27. Proper Kl, Koning M, van der Beek AJ, Hildebrandt VH, Bosscher RJ, van Mechelen $\mathrm{W}$. The effectiveness of worksite physical activity programs on physical activity, physical fitness, and health. Clin J Sport Med. 2003;13:106-17.

28. World Health Organisation. Ottawa charter for health promotion. Can J Public Health. 1986;77:425-30.

29. Pedersen MM, Zebis MK, Langberg H, Poulsen OM, Mortensen OS, Jensen JN et al. Influence of Self-Efficacy on Compliance to Workplace Exercise. IJBM. 2012;20(3):365-70.

30. Andersen LL, Saervoll CA, Mortensen OS, Poulsen OM, Hannerz H, Zebis MK Effectiveness of small daily amounts of progressive resistance training for frequent neck/shoulder pain: randomised controlled trial. Pain. 2011;152:440-6.

31. Bredahl TVG, Saervoll C, Kirkelund L, Sjøgaard G, Andersen LL. When Intervention Meets Organisation, a Qualitative Study of Motivation and Barriers to Physical Exercise at the Workplace. World Scientific J. 2014;2015: 12.(Workplace Health Promotion and Wellbeing).

32. Roessler KK, Rugulies R, Bilberg R, Andersen LL, Zebis MK, Sjogaard G. Does work-site physical activity improve self-reported psychosocial workplace factors and job satisfaction? A randomized controlled intervention study. Int Arch Occup Environ Health. 2013;86:861-4.

33. Hagger MS, Chatzisarantis NLD. Intrinsic motivation and self-determination in exercise and sport. Champaign: Human Kinetics; 2007.

34. Ajzen I. The theory of planned behavior. Organ Behav Hum. 1991;50:179-211.

35. Ajzen I. Behavioral Interventions Based on the Theory of Planned Behavior. 2002. http://citeseerx.ist.psu.edu/viewdoc/download?doi=10.1.1.613. 1749\&rep=rep1\&type=pdf. Accessed 15 Jul 2016

36. Kautiainen S, Koivisto AM, Koivusilta L, Lintonen T, Virtanen SM, Rimpela A. Sociodemographic factors and a secular trend of adolescent overweight in Finland. Int J Pediatr Obes. 2009;4:360-70.

37. Marques-Vidal P, Bovet P, Paccaud F, Chiolero A. Changes of overweight and obesity in the adult Swiss population according to educational level, from 1992 to 2007. BMC Public Health. 2010;10:87.

38. Emmons KM, Linnan L, Abrams D, Lovell HJ. Women who work in manufacturing settings: factors influencing their participation in worksite health promotion programs. Women's Health Issues: official Publication of the Jacobs Institute of Women's Health. 1996;6:74-81.

39. Harris JR, Huang Y, Hannon PA, Williams B. Low-socioeconomic status workers: their health risks and how to reach them. J Occup Environ Med. 2011:53:132-8

40. Sjogaard G, Justesen JB, Murray M, Dalager T, Sogaard K. A conceptual model for worksite intelligent physical exercise training-IPET-intervention for decreasing life style health risk indicators among employees: a randomized controlled trial. BMC Public Health. 2014;14:652.

41. Hussey MA, Hughes JP. Design and analysis of stepped wedge cluster randomized trials. Contemp Clin Trials. 2007;28:182-91.

42. Mdege ND, Man MS, Taylor Nee Brown CA, Torgerson DJ. Systematic review of stepped wedge cluster randomized trials shows that design is particularly used to evaluate interventions during routine implementation. J Clin Epidemiol. 2011;64:936-48.

43. Torgerson D. Designing Randomised Trials in Health, Education and the Social Sciences: An Introduction: Palgrave Macmillan UK; 2008

44. Brown CA, Lilford RJ. The stepped wedge trial design: a systematic review. BMC Med Res Methodol. 2006;6:54

45. May CR, Mair F, Finch T, MacFarlane A, Dowrick C, Treweek S, et al. Development of a theory of implementation and integration: Normalization Process Theory. IS. 2009:4:29.

46. Azadbakht L, Surkan PJ, Esmaillzadeh A, Willett WC. The Dietary Approaches to Stop Hypertension eating plan affects $C$-reactive protein, coagulation abnormalities, and hepatic function tests among type 2 diabetic patients. J Nutr. 2011:141:1083-8.

47. Basiotis PP, Welsh SO, Cronin FJ, Kelsay JL, Mertz W. Number of days of food intake records required to estimate individual and group nutrient intakes with defined confidence. J Nutr. 1987;117:1638-41. 
48. Nordic Nutrition Recommendations 2004: Integrating Nutrition and Physical Activity. Copenhagen: Nordic Council of Ministers; 2005

49. Linton SJ, Andersson T. Can chronic disability be prevented? A randomized trial of a cognitive-behavior intervention and two forms of information for patients with spinal pain. Spine. 2000;25:2825-31.

50. Linton SJ, Bradley LA, Jensen I, Spangfort E, Sundell L. The secondary prevention of low back pain: a controlled study with follow-up. Pain. 1989;36:197-207.

51. Saltin B, Grimby G. Physiological analysis of middle-aged and old former athletes. Comparison with still active athletes of the same ages. Circulation. 1968;38:1104-15.

52. Grimby G, Borjesson M, Jonsdottir $\mid H$, Schnohr $P$, Thelle DS, Saltin B. The "Saltin-Grimby Physical Activity Level Scale" and its application to health research. Scand J Med Sci Sports. 2015;25 Suppl 4:119-25.

53. Ware Jr JE, Sherbourne CD. The MOS 36-item short-form health survey (SF-36). Conceptual framework and item selection. Med Care. 1992;30:473-83.

54. Bjørner JB, Damsgaard MT, Watt T, Bech P, Rasmussen NK, Kristensen TS, et al. Dansk manual til SF-36: et spørgeskema om helbredsstatus. (Danish manual to SF-36: a questionnaire conserning health status). Copenhagen: Lif; 1997.

55. Marcus BH, Selby VC, Niaura RS, Rossi JS. Self-efficacy and the stages of exercise behavior change. Res Q Exerc Sport. 1992;63:60-6.

56. Sarkin JA, Johnson SS, Prochaska JO, Prochaska JM. Applying the transtheoretical model to regular moderate exercise in an overweight population: validation of a stages of change measure. Prev Med. 2001; 33:462-9.

57. Cohen S, Kamarck T, Mermelstein R. A global measure of perceived stress. J Health Soc Behav. 1983;24:385-96.

58. Kuorinka I, Jonsson B, Kilbom A, Vinterberg H, Biering-Sorensen F, Andersson G, et al. Standardised Nordic questionnaires for the analysis of musculoskeletal symptoms. Appl Ergon. 1987;18:233-7.

59. Labriola M, Lund T. Self-reported sickness absence as a risk marker of future disability pension. Prospective findings from the DWECS/DREAM study 1990-2004. Int J Med Sci. 2007;4:153-8.

60. Miller RP, Kori SH, Todd DD. The Tampa Scale. Unpublished Report: Tampa, FL, 1991

61. Tuomi K, Ilmarinen J, Jahkola A, Katajarinne L, Tulkki A. Work ability index. Helsinki: Finnish Institute of Occupational Health; 1998.

62. Borg G. Perceived exertion as an indicator of somatic stress. Scand J Rehabil Med. 1970;2:92-8

63. Kessler RC, Barber C, Beck A, Berglund P, Cleary PD, McKenas D, et al. The World Health Organization Health and Work Performance Questionnaire (HPQ). J Occup Environ Med. 2003;45:156-74.

64. Brouwer WB, Koopmanschap MA, Rutten FF. Productivity losses without absence: measurement validation and empirical evidence. Health Policy. 1999:48:13-27.

65. Jorgensen MB, Skotte JH, Holtermann A, Sjogaard G, Petersen NC, Sogaard K. Neck pain and postural balance among workers with high postural demands a cross-sectional study. BMC Musculoskelet Disord. 2011;12:176.

66. Glasgow RE, Vogt TM, Boles SM. Evaluating the public health impact of health promotion interventions: the RE-AIM framework. Am J Public Health. 1999;89:1322-7.

67. The Copenhagen City Heart Study Group. Osterbroundersogelsen. A book of tables with data from the first examination (1976-78) and a five year follow-up (1981-83). Scand J Soc Med. 1989:41:1-160.

68. Physical status: the use and interpretation of anthropometry. Geneva: World Health Organisation; 1995

69. Essendrop M, Maul I, Laubli T, Riihimaki H, Schibye B. Measures of low back function: a review of reproducibility studies. Clin Biomech. 2002;17:235-49.

70. Schibye B, Hansen AF, Sogaard K, Christensen H. Aerobic power and muscle strength among young and elderly workers with and without physically demanding work tasks. Appl Ergon. 2001;32:425-31.

71. Fairfax AH, Balnave R, Adams RD. Variability of grip strength during isometric contraction. Ergonomics. 1995;38:1819-30.

72. Backman $E$, Johansson V, Hager B, Sjoblom P, Henriksson KG. Isometric muscle strength and muscular endurance in normal persons aged between 17 and 70 years. Scand J Rehabil Med. 1995;27:109-17.

73. Yates JW, Kamon E, Rodgers SH, Champney PC. Static lifting strength and maximal isometric voluntary contractions of back, arm and shoulder muscles. Ergonomics. 1980;23:37-47.
74. AJ, Trygg J, Gullberg J, Johansson Al, Jonsson P, Antti H, Marklund SL, Moritz T. Extraction and GC/MS Analysis of the Human Blood Plasma Metabolome. Anal Chem. 2005;77(24):8086-94.

75. Woertman W, de Hoop E, Moerbeek M, Zuidema SU, Gerritsen DL, Teerenstra S. Stepped wedge designs could reduce the required sample size in cluster randomized trials. J Clin Epidemiol. 2013;66:752-8.

76. Sjøgaard G, Christensen JR, Justesen JB, Murray M, Dalager T, Fredslund GH, et al. Exercise is more than medicine: The working age population's well-being and productivity. JSHS. 2016;5:159-65.

\section{Submit your next manuscript to BioMed Central and we will help you at every step:}

- We accept pre-submission inquiries

- Our selector tool helps you to find the most relevant journal

- We provide round the clock customer support

- Convenient online submission

- Thorough peer review

- Inclusion in PubMed and all major indexing services

- Maximum visibility for your research

Submit your manuscript at www.biomedcentral.com/submit 